\title{
Metadata to the MARS spatial database
}

Lidija Globevnik, Maja Koprivsek \& Luka Snoj
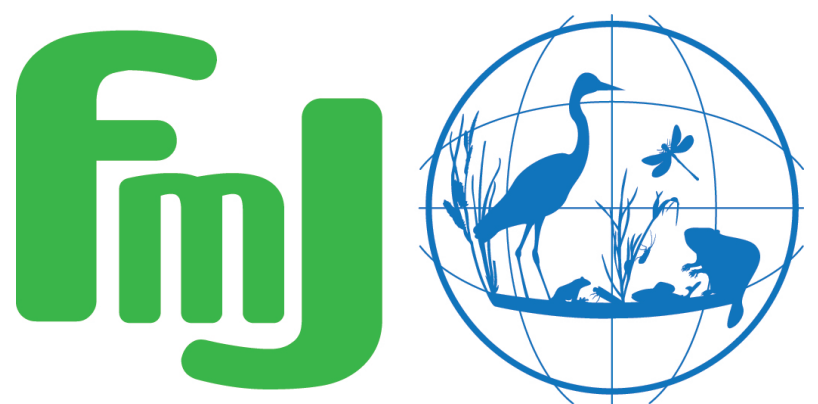

Freshwater Metadata Journal 



\title{
Metadata to the MARS spatial database
}

\author{
Lidija Globevnik ${ }^{1}$, Maja Koprivsek ${ }^{1}$ \& Luka Snoj $^{1}$ \\ 1 University of Ljubljana, Faculty of Civil and Geodetic Engineering, Ljubljana, Slovenia; corresponding author: \\ lidija.globevnik@fgg.uni-lj.si
}

Please cite this paper as follows: Globevnik L., Koprivsek M. \& Snoj L., 2017. Metadata to the MARS spatial database. Freshwater Metadata Journal 21: 1-7. https://doi.org/10.15504/fmj.2017.21

Received: 2017-01-20 / Published: 2017-03-06

\section{Keywords}

watershed characteristics, rivers/streams, lakes/reservoirs, ground water, ecological status, water quality/water chemistry, discharge/flow, land use/land cover, population density, nutrient load, climate characteristics

\section{Short description of the dataset/summary}

The MARS spatial database (MARSgeoDB) supports analyses of European waters, providing common reference spatial layers and selected data on indicators of pressures, state and impacts of European waters. It is developed within the European research project MARS (Managing Aquatic ecosystems and water Resources under multiple Stress) in accordance with the WISE (Water Information System in Europe) concept. It is built on the ECRINS (European Catchments and Rivers Network System) spatial database (from the European Environment Agency), consisting of river segments, lakes and functional elementary catchments (FECs). It includes other available European spatial layers, such as River Basin Districts (RBDs), RBD sub-units, coastlines, regions, water bodies as reported under the WFD (Water Framework Directive) in 2010 and WISE SoE (State of Environment) locations.

For spatial objects representing waters in the MARSgeoDB we compiled indicators of pressure, state and impact: physical-chemical indicators, ecological quality ratio, ecological status, chemical status, hydromorphological status, land use, population, nitrogen and phosphorus diffuse pollution, Eurostat agricultural data, UWWTD (Urban Waste Water Treatment Directive) point sources of organic pollution, E-PRTR (The European Pollutant Release and Transfer Register) point sources of large emissions to water, hydro-morphological changes/naturalness of rivers, meteorological and hydrological characteristics. To calculate pressures acting on selected locations on waters we derived surface water receiving areas (polygons representing catchments/hinterlands). We assigned broad ecological types to rivers (20 types) and lakes (15 types) objects in the MARSgeoDB using abiotic criteria as proposed by EEA ETC/ICM (European Topic Centre on Inland, Coastal and Marine waters) in 2015. A corresponding water body code and national ecological types were assigned as well.

Spatial and associated attribute data were quality checked, unified when needed, harmonised and interlinked. 


\section{General information}

dataset entry ID:

name of the dataset:

full name of the dataset:

dataset short name:

type of dataset:

data type:
MARS_20

MARS spatial database

MARSgeoDB

environmental characteristics database

vector data (shape files)

science keywords according to GCMD:

topic:

Agriculture, Biological Classification, Climate Indicators, Land Surface,

Terrestrial Hydrosphere

keywords:

ISO topic category according to ISO 19115:

DPSIR, WFD, WISE SoE, watershed characteristics, rivers/streams,

lakes/reservoirs, ground water, ecological status, water quality/water chemistry, discharge/flow, land use/land cover, population density, precipitation, air temperature, agriculture production

Farming, Boundaries, Climatology/Meteorology/Atmosphere, Elevation, Environment, Inland Waters

\section{Technical and administrative specifications}

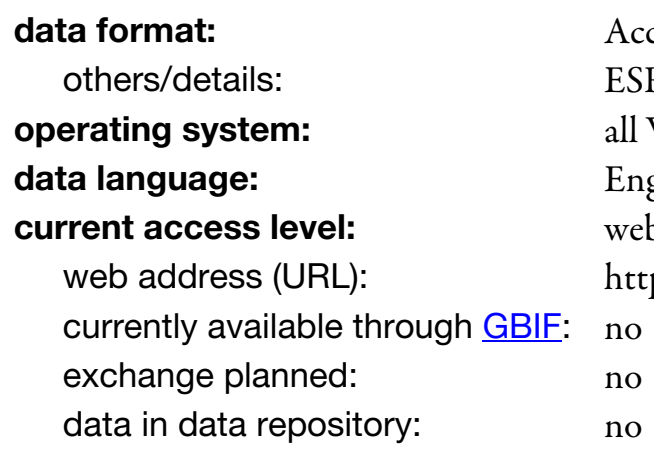

Do you plan to publish the data on the Freshwater Biodiversity Data Portal:

\section{update level:}

documentation:

type:

language:

contact details:

metadata contact person:

first, last name:

phone:

email:

institution:

address:

postal code, city:

country

web address:

technical contact person:

first, last name:

phone:

email: no

completed

manual

English

Lidija Globevnik

+386-41-738623

lidija.globevnik@fgg.uni-lj.si

University of Ljubljana, Faculty of Civil and Geodetic Engineering

Jamova 2

1000 Ljubljana

Slovenia

https://www.uni-lj.si/academies_and_faculties/faculties/2013071111381151/

Lidija Globevnik

$+386-41-738623$

lidija.globevnik@fgg.uni-lj.si 
scientific contact person:
first, last name:
Lidija Globevnik
phone:
+386-41-738623
email:
lidija.globevnik@fgg.uni-lj.si

\section{Intellectual property rights and citation}

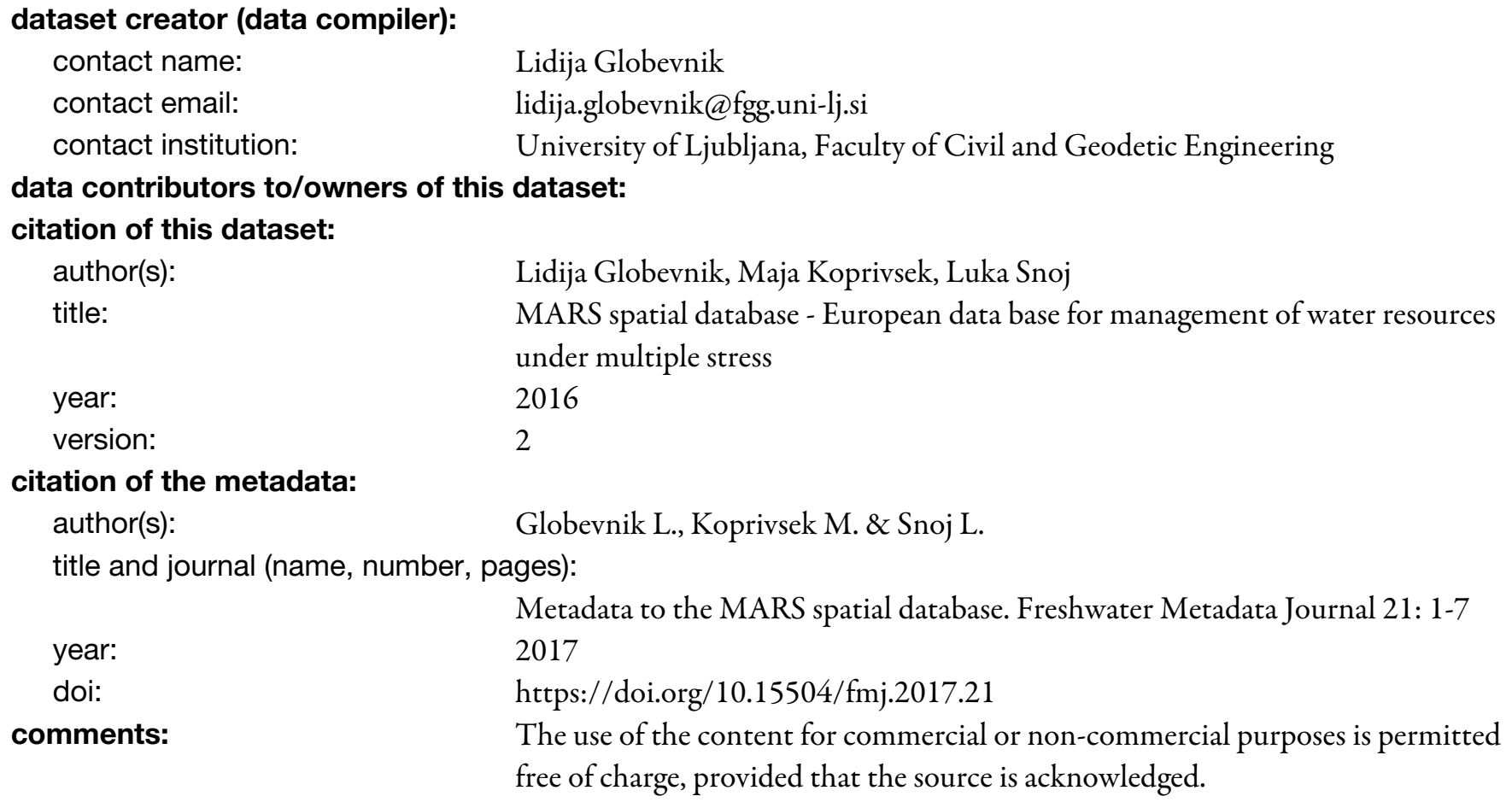

Metadata to the MARS spatial database. Freshwater Metadata Journal 21: 1-7

year: 2017

doi: https://doi.org/10.15504/fmj.2017.21

comments:

The use of the content for commercial or non-commercial purposes is permitted free of charge, provided that the source is acknowledged.

\section{General data specifications}

\section{regional coverage of the dataset:}

scale of the dataset:

continents:

spatial extent (bounding coordinates):

southernmost latitude $\left[{ }^{\circ}\right]$ :

northernmost latitude $\left[^{\circ}\right]$ :

westernmost longitude $\left[^{\circ}\right]$ :

easternmost longitude [ $\left.{ }^{\circ}\right]$ :

minimum altitude:

maximum altitude:

countries:

comments: continental

Europe

33.727485

71.185599

$-24.533308$

42.642135

-10 metres

4442 metres

Europe: Åland Islands, Albania, Andorra, Austria, Belarus, Belgium, Bosnia and Herzegovina, Bulgaria, Croatia, Czech Republic, Denmark, Estonia, Finland,

France, Germany, Gibraltar, Greece, Guernsey, Hungary, Iceland, Ireland, Isle of Man, Italy, Latvia, Liechtenstein, Lithuania, Luxembourg, Macedonia, Malta, Moldova, Monaco, Montenegro, Netherlands, Norway, Poland, Portugal, Romania, Russia, San Marino, Serbia, Slovakia, Slovenia, Spain, Sweden, Switzerland, Ukraine, United Kingdom, Vatican City, Kosovo EU-28 + NO, IS, CH, LI, AD, RS, BA, AL, MK, ME and XK + Turkey (without Euphrates and Tigris River basins) + part of Syria and Lebanon (Asi River basin) + parts of Russia (Pregolya, Daugava, Neva, Oulujoki, Kovda and 
world climatic regions according to Köppen:

Lotta River basins), Belarus (Daugava, Neman, Vistula River basins), Ukraine

(Danube and Vistula River basins), Moldova (Danube River basin)

Some layers (feature classes) are not covering all the countries listed above.

Group B: dry (arid and semiarid) climates

Group C: temperate/mesothermal climates

Group D: continental/microthermal climate

Group E: polar climates

Group $\mathrm{H}$ : alpine climates

freshwater ecoregions of the world (FEOW) according to WWF:

Europe: Aegean Drainages, Barents Sea Drainages, Cantabric Coast -

Languedoc, Central \& Western Europe, Central Anatolia, Dalmatia, Dniester Lower Danube, Eastern Iberia, Gulf of Venice Drainages, Iceland - Jan Mayen, Ionian Drainages, Italian Peninsula \& Islands, Lake Onega - Lake Ladoga, Northern Anatolia, Northern Baltic Drainages, Northern British Isles, Norwegian Sea Drainages, Orontes, Southeastern Adriatic Drainages, Southern Anatolia, Southern Baltic Lowlands, Southern Iberia, Thrace, Upper Danube, Vardar, Western Anatolia, Western Iberia, Western Transcaucasia

\section{European ecoregions according to Illies (WFD):}

Iberic-Macaronesian Region (ER1), Pyrenees (ER2), Italy, Corsica and Malta (ER3), Alps (ER4), Dinaric Western Balkan (ER5), Hellenic Western Balkan (ER6), Eastern Balkan (ER7), Western Highlands (ER8), Central Highlands (ER9), The Carpathians (ER10), Hungarian Lowlands (ER11), Pontic Province (ER12), Western Plains (ER13), Central Plains (ER14), Baltic Province (ER15), Eastern Plains (ER16), Ireland and Northern Ireland (ER17), Great Britain (ER18), Iceland (ER19), Borealic Uplands (ER20), Tundra (ER21), Fenno-Scandian Shield (ER22), Taiga (ER23), The Caucasus (ER24)

ecosystem type: comments:

\section{Site specifications}

coordinate system/grid data: datum (e.g. WGS84):

grid data available:

resolution:

unit:

comments:

number of sites: comments: projected, others others: ETRS89_LAEA

D_ETRS_1989

yes

1

$\mathrm{km}$

Grid data are available for climatological data, land cover data, altitude as well as slope, population density and population count. Data of different spatial resolutions are resampled on $1 \mathrm{~km}$ grid.

$>1000$

There are different numbers of sites in different layers (feature classes), for example: 16694 WISE SoE rivers quality stations, 26794 UWWTD discharge points, 5043 dams, 15016 E-PRTR facility report points. All compiled data have been linked to the ECRINS catchment and river network system when possible. 


\section{Climate and environmental data}

climate related data:

spatial resolution of the data (if not catchment/site related):

$1 \mathrm{~km}$

others: $\quad$ Data are available per catchment (FEC and hinterland) and in grid (in different original resolutions depending on the source and resampled to $1 \mathrm{~km}$ grid).

available parameters per catchment:

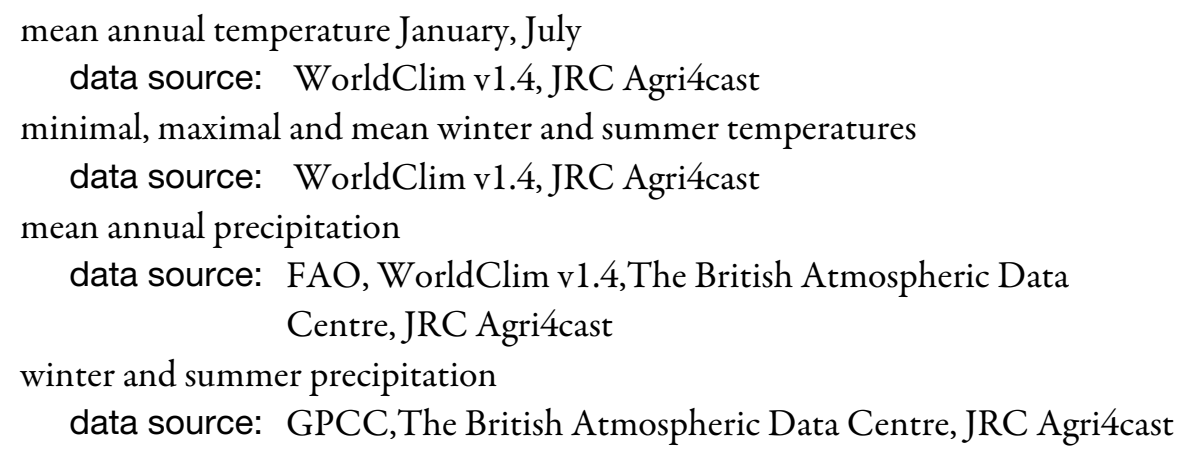

\section{environmental data:}

available parameters per catchment:

catchment size

data source: ECRINS v1.1

catchment geology

data source: BGR - IHME 1500_v11, JRC - SGDBE4, WFD reporting, WRc

catchment land cover/land use

data source: CLC2006 v17, CLC2000Greece, GlobCorine2009, EEA

Copernicus land cover/land use

population density

data source: EEA, Population density disaggregated with CLC2000,

SEDAC Gridded Population v3

presence of barriers/dams/reservoirs (fragmentation)

data source: ECRINS v1.1, ESRI basemap

hydrological regime/flow regime

data source: PCR-GLOBWB (DELTARES, NL)

available parameters per site:

catchment land use upstream of sampling site

data source: CLC2006 v17, CLC2000Greece, GlobCorine2009, EEA

Copernicus land cover/land use

catchment land use along a buffer strip (100m width on both sides) upstream

$(10 \mathrm{~km})$ of the sampling site

data source: CLC2006 v17, CLC2000Greece, GlobCorine2009,EEA

Copernicus land cover/land use

river length

data source: ECRINS v1.1

distance to source

data source: ECRINS v1.1

distance to mouth

data source: ECRINS v1.1

stream order (according to Strahler)

data source: ECRINS v1.1 


\section{physico-chemistry data:}

other physico-chemical parameters:

chemical oxygen demand, dissolved organic carbon, dissolved oxygen, Kjeldahl nitrogen, silicate

availability of physico-chemical data, if there is more than one sample per site:

mean values per site

comments:

stressors influencing the sites:

reference sites available:

\begin{tabular}{|c|c|c|c|c|}
\hline stressor & $\begin{array}{l}\text { restored sites } \\
\text { available }\end{array}$ & $\begin{array}{l}\text { data before/after } \\
\text { restoration } \\
\text { available }\end{array}$ & $\begin{array}{l}\text { stressor gradient } \\
\text { available }\end{array}$ & comments \\
\hline eutrophication & no & no & no & $\begin{array}{l}\text { TotP, total N, orthophoshate } \\
\text { concentrations }\end{array}$ \\
\hline $\begin{array}{l}\text { hydromorphological } \\
\text { degradation }\end{array}$ & no & no & no & $\begin{array}{l}\text { alteration of natural riparian } \\
\text { habitats }\end{array}$ \\
\hline organic pollution & no & no & no & $\begin{array}{l}\text { represented by BOD5, } \\
\text { ammonium and nitrates }\end{array}$ \\
\hline general degradation & no & no & no & $\begin{array}{l}\text { EQR of invertebrates, EQR of } \\
\text { macrophytes }\end{array}$ \\
\hline $\begin{array}{l}\text { hydrologic stress } \\
\text { (e.g. impoundment, } \\
\text { flow velocity } \\
\text { reduction, } \\
\text { hydropeaking, water } \\
\text { abstraction, flow } \\
\text { velocity increase) }\end{array}$ & no & no & no & $\begin{array}{l}\text { flow alteration ratio } \\
\text { (abstraction/no abstraction) }\end{array}$ \\
\hline
\end{tabular}

comments:

Proxy stressors for eutrophicaton are also: 1) share of agricultural land in catchment (upstream drainage area), in local drainage area ( $\mathrm{FEC}=$ functionally elementary catchment) and along the river (buffer/strip area), 2) level of urban waste water treatment, 3) population density and 4) data on agricultural activities such are total yearly input of $\mathrm{N}$ and $\mathrm{P}$ (tonnes/year).

\section{Other specifications}

\section{GIS layers, shapes related to the dataset:}

hydrological information (as HydroSHEDS)

catchments, river-sub-basins

land use 
others (specify):

\section{availability of photos: availability of maps: quality control procedures: \\ no \\ yes}

Were any quality control procedures applied to your dataset?

yes dams/reservoirs/barriers

protected areas

population density

environmental variables (freshwater or terrestrial)

climatic variables (current and predictions)

others/specify

polygons: EUROSTAT NUTS, country borders, coastal line, WFD ecoregions (Illies), biogeographical regions (EEA, Habitat Directive), broad hydroregions (IC fish), hydro ecoregions (Rebecca project), WWF hydro regions point objects: WFD surface water bodies (2010), WFD groundwater bodies (2010) WISE SoE stations, EFI+ stations

quality control protocols and comments:

When linking point pressure/state data to ECRINS hydrological catchments and river network data, spatial quality checks were performed as well as attributive QA checks (river name check, (sub)catchment check).

\section{Acknowledgements}

This work was funded by the MARS project (Managing Aquatic ecosystems and water resources under multiple stress), funded by the European Union under the 7th Framework Programme, contract no. 603378.

\section{References}

Daniel Hering, Laurence Carvalho, Christine Argillier, Meryem Beklioglu, Angel Borja, Ana Cristina Cardoso, Harm Duel, Teresa Ferreira, Lidija Globevnik, Jenica Hanganu, Seppo Hellsten, Erik Jeppesen, Vit Kodes, Anne Lyche Solheim, Tiina Nõges, Steve Ormerod, Yiannis Panagopoulos, Stefan Schmutz, Markus Venohr, Sebastian Birk, 2015. Managing aquatic ecosystems and water resources under multiple stress - An introduction to the MARS project https://doi.org/10.1016/j.scitotenv.2014.06.106

ETC/ICM, 2015. European Freshwater Ecosystem Assessment: Cross-walk between the Water Framework Directive and Habitats Directive types, status and pressures. ETC/ICM Technical Report 2/2015. Magdeburg: European Topic Centre on inland, coastal and marine waters, 95 pp. plus Annexes. 
Appendix 


\section{Example layer from MARS spatial database}

Figure below shows layer of FECs coloured by broad river type. Each FEC has been assigned one representative river broad type (delegated from a river segment that represents FEC outflow). Number in legend represents broad river type as defined by ETC/ICM (2015).

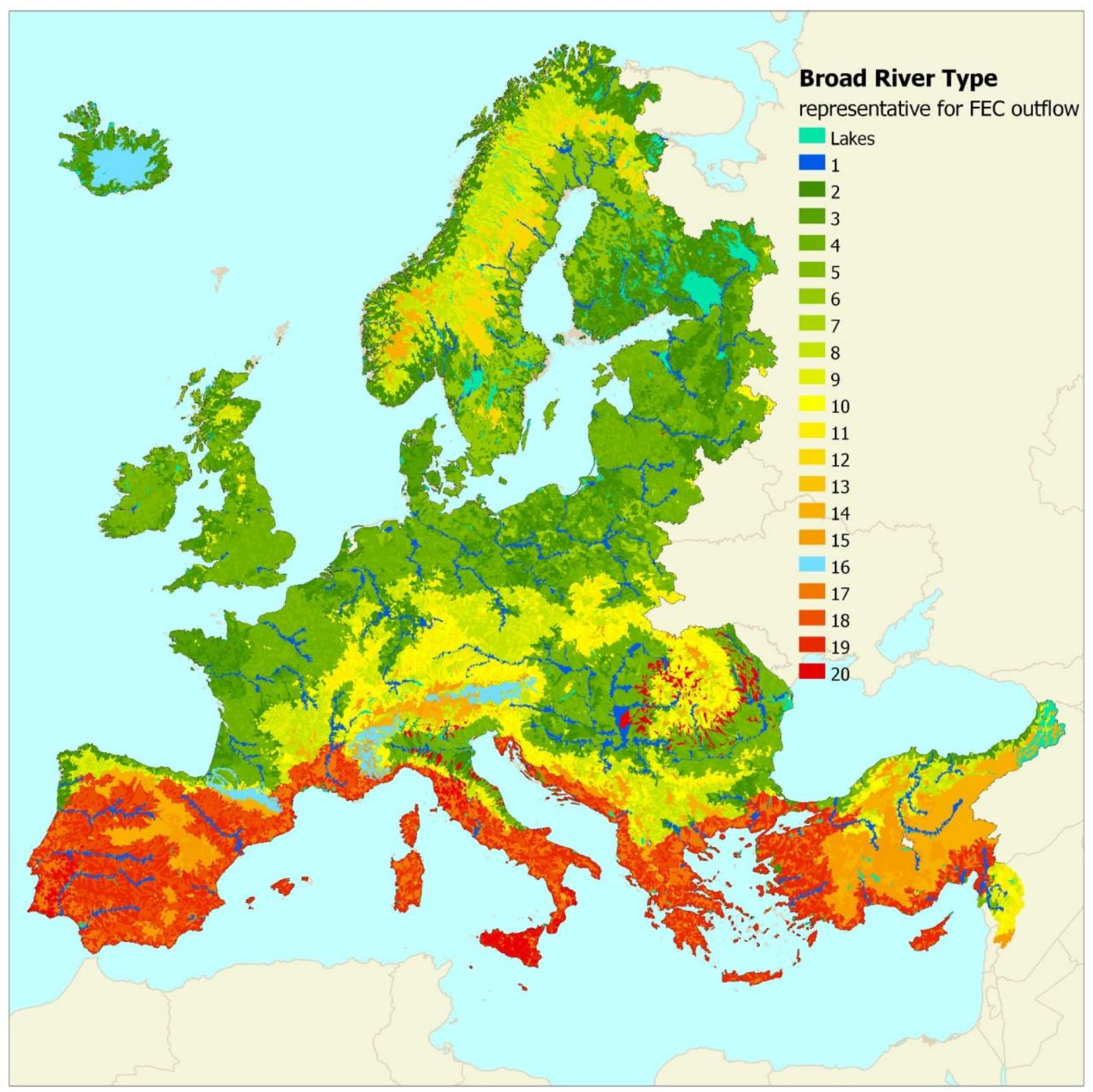

